\title{
FRINGE VISIBILITY AND PHASE MEASUREMENTS
}

\author{
M. SHAO \\ Jet Propulsion Laboratory \\ California Institute of Technology \\ 4800 Oak Grove Dr., Pasadena, CA 91109, USA
}

\begin{abstract}
.
At optical wavelengths there are a wide variety of techniques to detect interference fringes. Because detectors in the visible/IR are energy detectors, the fringe amplitude and phase must be encoded in some manner. Two fundamentally different methods are temporal and spatial encoding. This paper briefly describes the various techniques that have been used in operational interferometers along with their advantages and disadvantages. Several fringe detectors for nextgeneration interferometers will also be described. These fringe detectors often combine multiple functions, such as spectroscopy and multi-beam combination.
\end{abstract}

\section{Introduction}

This paper reviews the evolution of fringe detection techniques used in optical and IR interferometry. In O/IR interferometry, interference is usually detected by directly combining the stellar radiation on a detector. In the radio, in contrast, the stellar radiation is usually detected at each telescope with a detector that preserves phase, and is then distributed to correlators to measure the interference between the signals.

The next section of this paper discusses the fundamental difference between radio and $\mathrm{O} / \mathrm{IR}$ interferometry and the advantages/disadvantages of using radio techniques in the $\mathrm{O} / \mathrm{IR}$, and $\mathrm{O} / \mathrm{IR}$ techniques at radio wavelengths. With this background, the rest of this paper then describes the various techniques for fringe detection in use or under development for O/IR interferometry.

\section{Radio vs. O/IR Interferometry}

The fundamental difference between radio and O/IR interferometry is the use of energy detectors in $\mathrm{O} / \mathrm{IR}$ and electric field detectors in the radio. At radio wavelengths, the existence of electric field detectors and nearly noise-free amplifiers means that as one increases the number of telescopes, the number of baselines, over which interferometric information is being obtained, goes as $N(N-1) / 2$. In the optical, the light from each telescope must be split $N-1$ ways before the $N(N-1) / 2$ baseline measurements can be made.

This apparent advantage of radio techniques has led many in radio astronomy to wonder if advances in technology to sub-mm and shorter wavelengths would ultimately mean that radio techniques would be adopted at optical/IR wavelengths. However, a more careful look at the fundamental limits of radio detection techniques (quantum noise of $h \nu$ per $\mathrm{Hz}$ of bandwidth) shows that even if the technology could be extended to shorter wavelengths, traditional O/IR techniques would provide significant advantages in sensitivity.

In the detection of astronomical radiation there are two generic sources of noise: additive noise and multiplicative noise. Additive noise is noise that is present even 
when there is no signal. Multiplicative noise is noise that is dependent on signal strength, usually as the square root of the signal strength.

At O/IR wavelengths, examples of additive noise include the dark count of a photon-counting detector, the thermal background of the optics at IR wavelengths, or sky background at O/IR wavelengths. The principle example of multiplicative noise is photon statistics (from the source). Because of the need to make fringe measurements in a short period of time at O/IR wavelengths $(\sim 10 \mathrm{~ms}$ at $0.55 \mu \mathrm{m})$, photon noise is usually the dominant noise source. It is convenient to think of noise as a process that occurs at the detector.

At radio wavelengths, additive noise dominates except perhaps for the brightest sources. Examples of additive noise are thermal background, detector noise, and quantum noise. However, multiplicative noise, i.e., photon noise from the source, is also present. Under ideal conditions, in the radio, the detector is perfect, there is no background, and the only source of additive noise is quantum noise. One way to understand quantum noise is to think of it as the shot noise/photon noise from the local oscillator in the heterodyne system. At visible wavelengths the bandwidth of the detector may be $400 \mathrm{THz}$. A heterodyne receiver at the quantum limit would have additive noise comparable to a photon-counting detector with a dark count of $4 \times 10^{14}$ counts per second. So while the lack of optical amplifiers that could be used to amplify the stellar signal prior to splitting the light from each telescope $N-1$ ways has been cited as a disadvantage of O/IR techniques, the quantum noise of such phase-preserving amplifiers would actually make the SNR many orders of magnitude worse.

\section{O/IR Fringe Detectors: I. Amplitude and Phase}

While optical interferometry dates back to Michelson, decades before the birth of radio astronomy, interferometry at radio wavelengths is much more developed. In the early years of optical interferometry, fringes were detected by eye. While the eye in many respects is a very good detector, it lacks the ability to make quantitative measurements. By the time technology had progressed to the point where multipletelescope long-baseline O/IR interferometer were feasible, electronic detectors had replaced the eye.

The early detectors were single-element (not imaging) detectors. Fringes, however, are sinusoidal variations in the intensity of light with changing optical path. These sinusoidal variations then had to be temporally encoded onto one detector. Two methods have been used on operational interferometers to date. One technique varies the optical path by many microns, scanning the whole fringe packet. In this way the spectrum of the star is simultaneously measured. The second technique varies the optical path by one wavelength while the sinusoidal change in intensity is recorded.

Scanning the whole fringe packet has a SNR disadvantage. Typically the fringe measurement has to be made within $\tau_{0}$, the atmospheric coherence time. Only the central fringe of the packet has high visibility, whose reduction is due to a resolved star. In the photon and background noise limited regime, the disadvantage of the packet vs. single wavelength scanning in sensitivity is as the ratio of the scan lengths. 
On the other hand, scanning a single fringe places additional requirements on the operation of the interferometer. Each fringe in the fringe packet has a different amplitude. If only one of the fringes are scanned, then the interferometer must be able to identify which fringe it is scanning in order for the visibility measurement to be accurate. Two methods are used to ensure the correct visibility is being measured. The first is to use a narrow bandwidth so that visibility of several adjacent fringes are not significantly $(\sim 1 \%)$ different. The second is to collect data at several wavelengths simultaneously and use that information to identify and track the central fringe.

In astrometry, the position (phase) of the central fringe is related to the position of the star as

$$
x=\mathbf{B} \cdot \hat{s}+C,
$$

where $x$ is the fringe position, $\mathbf{B}$ is the baseline vector, $\hat{s}$ is a unit vector to the star, and $C$ is a constant (delay offset) that is dependent on the zero point of the laser metrology system. In astrometry there is a need to uniquely identify the fringe; otherwise the fringe position $x$ can have several values differing by $N \lambda$.

\section{O/IR Fringe Detectors: II. Multispectral Detectors}

Because identification of the central fringe is important, and for numerous other reasons, a number of current interferometer projects are designing and building beam combiners that measure the fringe visibility and phase at many wavelengths simultaneously. These multispectral fringe detectors are being built in most cases with multi-pixel/imaging detectors. The typical design is a low-resolution dispersive spectrometer with a linear array. The spectrometer is placed after the light has been combined from the two or more telescopes.

With data from multiple adjacent spectral channels, several types of information can be derived that were not easily obtainable before. One is the group delay-the position of the central fringe. The group $g$ and phase $\phi$ delay are related by

$$
g=\frac{\mathrm{d} \phi}{\mathrm{d} \nu}
$$

where $\nu=1 / \lambda$. Thus, the change in the fringe phase with frequency uniquely defines the group delay or the position of the central fringe.

If the number of spectral channels is large, the group delay can be derived directly from the output of the spectrometer, without fringe modulation/demodulation to make phase/amplitude measurements at each spectral channel. If the path difference between the two arms of the interferometer is, for example, $3 \mu \mathrm{m}$, then there is constructive interference at $0.5 \mu \mathrm{m}$ ( 6 waves) and $0.6 \mu \mathrm{m}$ ( 5 waves) and destructive interference at $0.55 \mu \mathrm{m}$ ( 5.5 waves) and $0.67 \mu \mathrm{m}$ ( 4.5 waves). A Fourier transform of the spectrum is, to first order, a delta function at the group delay.

The other reason for building multispectral fringe detectors is to be able to measure the diameters of stars in spectral lines. 


\section{O/IR Fringe Detectors: III. Multiple Beam Combiners}

To date, all long-baseline O/IR interferometers have been two-element systems. However, several projects are under way that will in the coming one or two years combine the light from three or more telescopes. Going from one baseline to three or more baselines increases the complexity of the beam combiner by at least a factor of three. Multiple simultaneous baselines is an important step for O/IR interferometry. One reason is that the closure phase can be measured. Phase information is normally seriously corrupted by atmospheric turbulence and closure phase is one of the few techniques that let us measure true phase. Another reason multiple baselines are important is for the calibration of amplitude measurements.

As the number of telescopes increase, the number of baselines grows as $N(N-$ 1)/2. Multiple beam combiners must accommodate this increase in information. One approach being used for 3-4 element beam combiners is to use beamsplitters to split the light from each telescope 2-3 ways. The light is then redirected to $N(N-1) / 2$ different beamsplitters to be recombined two beams at a time.

A second approach is to use beamsplitters to place all the beams on a single detector, but use different frequency path-length modulation on each beam so that the composite signal can be demodulated with unique signals for the $N(N-1) / 2$ baselines.

The third approach is to use an imaging/area detector. Multiple beams are directed at the detector at different angles. The angles are chosen such that the various pairs of combining beams form non-redundant spatial fringe patterns on the surface of the detector. With two-dimensional detectors, the second dimension can be used for spectroscopy.

Each of the various approaches have advantages and disadvantages. Some detectors, eg. CCD's and IR arrays, have a noise source associated with the number of times each pixel is read out. Photon- counting arrays have low QE. In all cases, the SNR for a single baseline decreases as the number of telescopes increase if the beam combiner produces outputs for all $N(N-1) / 2$ baselines. When coupled with the fact that fringe measurements must be made with telescopes of diameter $r_{0}$ or less, and with a coherent integration time of $\tau_{0}$ or less, the decrease in sensitivity with more telescopes severely limits the usefulness of multi-telescope interferometry.

The solution to this dilemma, at least in the IR, is described next.

\section{O/IR Fringe Detectors: IV. Multiple Object Combiners}

A technique used in the radio called phase-reference interferometry can be used at $\mathrm{O} / \mathrm{IR}$ wavelengths. In a manner similar to adaptive optics, the idea is to use the light from a "guide star" to phase the interferometer array. Once phased, integration time can be extended significantly beyond $\tau_{0}$. At $2.2 \mu \mathrm{m}, \tau_{0}$ is typically $50 \mathrm{~ms}$ : increasing $\tau_{0}$ to $500 \mathrm{~s}$ increases the sensitivity by a factor of 10,000 , or 10 stellar magnitudes.

Phase referencing in the O/IR is very different from its counterpart in the radio, and is much more similar to the use of guide stars in adaptive optics. Phase referencing is possible only if a sufficiently bright star is present within the isoplanatic angle of the desired target object. At visible wavelengths, the isoplanatic angle is 
only 2-3 arcsec, making the technique of marginal value. However, the isoplanatic angle increases as $\lambda^{1.2}$. Similarly, $r_{0}$ and $\tau_{0}$ also increase as the 1.2 power, and so the magnitude limit of the guide star gets fainter at longer wavelengths. The density of stars in the sky also increases as the magnitude gets fainter, almost linearly, i.e., a factor of 2 fainter means roughly twice as many stars. Taken all together, the fraction of the sky where there exists a guide star within the isoplanatic angle grows with wavelength as $\lambda^{6}$. Under excellent seeing conditions, phase referencing for long-baseline interferometers make sense at wavelengths longer than $\sim 2 \mu \mathrm{m}$, where a significant fraction of the sky (40-80\%) would have a guide star. When a guide star is available, phase referencing can increase sensitivity by a factor of $\sim 10,000$.

Phase referencing can be extended to shorter wavelengths by using individual telescopes larger than $r_{0}$ if there is a laser beacon system to phase the telescope. With laser guide star adaptive optics to phase a large aperture telescope $(\sim 8 \mathrm{~m})$, the phase-referencing relation now goes as $\lambda^{4}$-still a very steep function. Even with an array of $8 \mathrm{~m}$ telescopes, phase referencing would not work for wavelengths shorter than $\sim 1 \mu \mathrm{m}$. At shorter wavelengths higher sensitivity is possible only by going to space.

Multiple object beam combiners, because they are designed for phase-reference interferometry, must have accurate metrology to control the optical paths in the various parts of the interferometer. The optical path from each telescope to the point where the light from multiple telescopes is combined must be measured with $\lambda / 20$ accuracy for both the guide star and target object.

\section{Conclusion}

O/IR interferometry has made significant progress in the last 10 years. Starting with single spectral channel two-way beam combiners, O/IR interferometry in the 1990's is moving toward beam combiners that accommodate beams from 3-6 telescopes, have 10-100 spectral channels, and are potentially 10,000 times more sensitive than the first-generation interferometers built in the 1980's.

\section{Acknowledgements}

This work was performed at the Jet Propulsion Laboratory, California Institute of Technology, under a contract with the National Aeronautics and Space Administration.

\section{Discussion:}

Miller:

Multiple beams on a single detector brings up a method that was discussed in IR detection under the name of multiplex advantage. There is an advantage in total $\mathrm{S} / \mathrm{N}$ if detector noise dominates.

Shao:

The noise properties of the detector are an important and often driving factor in deciding what optical configuration should be used for beam combination. 


\section{Burke:}

There were two points in your talk that show the need for good communication between radio and optical interferometrists. (1) You remarked that in the radio, one measures voltage, and in the optical it is the energy that is measured. In fact, the same type of measurement is made in both regimes; both measure the cross-power spatial spectrum. (2) Bootstrapping the phase seems to have been reinvented; the method has long been used in radio aperture synthesis.

Shao:

At least we didn't waste much time reinventing bootstrapping!

\section{Buscher:}

It should be noted that beam recombination schemes which interfere all the beams onto one detector do not require closure-phase calibration. This applies both to spatially-sampled and temporally-sampled schemes.

Shao:

I agree that to first order these combiners don't need closure calibration. Second order effects may exist that would be important for high dynamic range imaging.

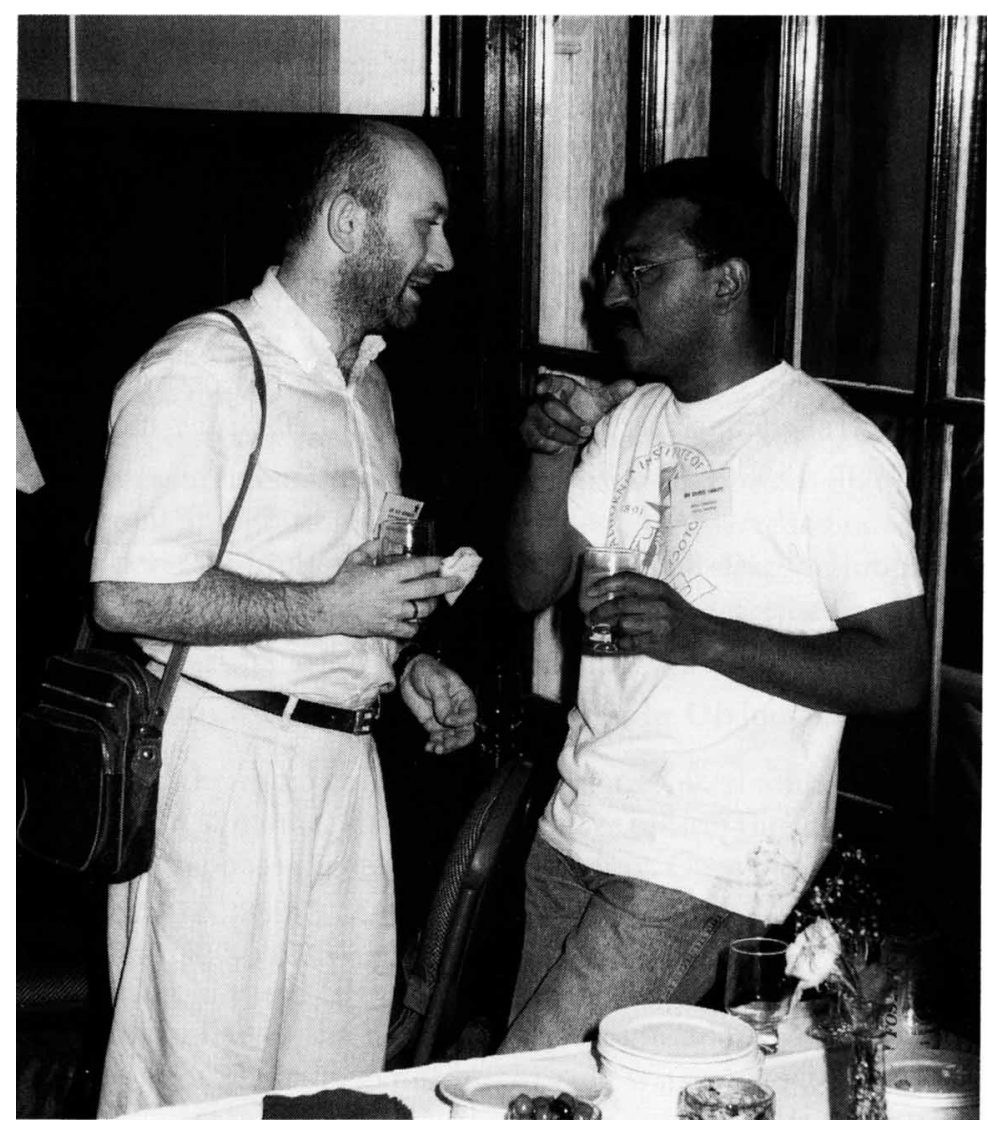

